\title{
Eye movements of monkeys during performance of ambiguous cue problems
}

\author{
N. D. GEARY and ALLAN M. SCHRIER \\ Brown University, Providence, Rhode Island 02912
}

\begin{abstract}
Eye movements of four stumptailed monkeys were measured during performance of simple and ambiguous form discriminations. Duration of fixations increased during learning of the simple discrimination, whereas the number of shifts in fixation from one stimulus to the other (scanning) increased and subsequently decreased. The asymptotic levels of scanning in both simple and ambiguous discriminations were consistent with predictions generated by a minimum frequency of observing hypothesis. Duration of fixations other than the last fixation preceding the choice response did not change significantly during performance of an ambiguous cue problem in comparison with the simple discrimination, but duration of the last fixation increased significantly. This result was discussed in terms of altered information processing requirements.
\end{abstract}

Visual observing responses, responses which expose the animal to the discriminative stimuli in visual discrimination tasks, are prerequisite to learning, but little is known about the functions of the various aspects of eye movements in this connection. Several experiments involving arbitrary, nonocular observing responses (such as pedal or keypresses) suggest that the number of observing responses declines after problem solution to a low level which might represent the minimum necessary for accurate performance (D'Amato, Etkin, \& Fazzaro, 1968; Premack \& Collier, 1966). In some studies of eye movements during discrimination learning, the number of scans, defined as the number of shifts in fixation from one stimulus to another, has declined to approximately the minimum necessary to fixate the positive cue (Schrier \& Wing, 1973; White \& Plum, 1964). Outcomes such as these have led some (Trabasso \& Bower, 1968) to suggest that observing behavior, or at least overt observing behavior, provides little information about discriminative and attentional processes. Is, however, minimum frequency of observing a general characteristic of the terminal visual behavior of animals in discrimination situations? Previous investigators have employed only simple two-choice discriminations, in which the amount of scanning required is very low. The minimum frequency of observing hypothesis might be more rigorously tested in a situation which requires higher minimum levels. This was achieved in the present experiment by measuring eye movements of stumptailed monkeys during performance of ambiguous cue discriminations as well as simple discriminations.

The ambiguous cue problem (French, 1965) is a conditional discrimination problem in which a given stimulus may be either positive or negative depending

This research was supported by NSF Research Grant GB-38580 to the second author. We are grateful to Dr. M. L. Povar, whose help throughout this research was invaluable. Requests for reprints should be addressed to the second author. upon the stimulus with which it is paired. The problems presented here involved successively more ambiguous cues, which required the animals to make successively greater numbers of visual fixations on the discriminative stimuli in order to locate the positive stimulus.

It has heen assumed that changes in information processing are directly reflected in the duration of fixations of humans (Gould, 1967; Neisser, 1963). On both theoretical and empirical grounds, the ambiguous cue problem would seem to require more information processing than simple discrimination problems. In this experiment, measurement of duration of fixation provided a test of the applicability of this assumption to monkeys' discrimination performance.

\section{METHOD}

\section{Subjects}

Four wild-born male stumptailed monkeys (Macaca arctoides), between 4 and 6 years of age, served as subjects. They were housed in individual cages in a colony room where they had free access to water. On test days, each animal received about $50 \%$ of its $140-\mathrm{g}$ daily food ration in the form of $190-\mathrm{mg}$ banana-flavored whole-diet pellets (Noyes, Lancaster, N. H.), which were used as reinforcers in the experimental situation, with the balance of the ration fed in the home cages. The animals' weights remained constant or increased very slightly during the course of the experiment. Two animals, Thad and Grady, were tested before the other two, Klaus and Hadyn. There were slight changes in procedure for the two groups, as described below.

\section{Apparatus}

The main components of the apparatus were: (a) a restraining chair, (b) a "crown" set on the monkey's head which could be fixed to the restraining chair so as to preclude head movements, and (c) trial presentation and data recording systems controlled by a computer.

During testing, the animal was seated in the restraining chair facing three stimulus-response devices mounted about $25 \mathrm{~cm}$ in front of its head. The devices were pigeon pecking keys (BRS/LVE, Beltsville, Md.), $2 \mathrm{~cm}$ in diam, arranged in the form of an inverted isosceles triangle. The translucent lower (center) key was separated from each of the transparent upper (side) keys 
by $10 \mathrm{deg}$ of visual angle and two side keys were separated from each other by $14 \mathrm{deg}$. The center key was illuminated with a small dot (about $.6 \mathrm{~mm}$ ) of light during a calibration phase of the trial preceding the discrimination phase. The animal viewed the discriminative stimuli, which were projected on the screens of Microminiature In-line display units (Industrial Electronic Engineers, Van Nuys, California, Model 340), through the side keys. The stimuli for Thad and Grady were a semicircle, an inverted $T$, and a right triangle which were of equal luminance (about $15.4 \mathrm{~cd} / \mathrm{m}^{2}$ ), color (red), and size (about $1 \mathrm{deg} 20 \mathrm{~min}$ of visual angle in width and $40 \mathrm{~min}$ in height). The stimuli for Klaus and Hadyn, a circle, a square, and a triangle, were brighter (about $21 \mathrm{~cd} / \mathrm{m}^{2}$ ) and smaller (about one-third the size) than those used for the other animals, and also were white.

The crown was modeled after the head restraint system developed by Freindlich (1973). It consisted of an aluminum rod bent around so that its ends could be welded together and shaped so as to conform roughly to the contours of the upper part of the monkey's head. With the animal anesthetized, seven stainless steel pressure screws were threaded through holes in the crown, passed through small slits in the skin of the scalp, and pressed against the skull. These crowns remained firmly in place for from 6 weeks to several months, after which they were removed for about 2 weeks and then repositioned. During experimental sessions, the crown was clamped to the restraining chair.

A LINC-8 computer (Digital Equipment Corporation, Maynard, Mass.) controlled the experimental events and recorded on magnetic tape the keypress responses and the location of a spot of light reflected from the cornea of the right eye which was sensed by a special TV camera (Massey Dickenson Co., Saxonville, Mass.). The computer recorded the location of the eye spot within a 15 -cell by 15 -cell recording grid 60 times/sec. Each cell of the grid represented approximately $1.5 \mathrm{deg}$ of visual angle. The computer interface has been described in detail in previous publications [Schrier, Povar, \& Vaughan $(1970,1971)]$.

\section{Procedure}

Testing sessions were run daily, 5 to 7 days a week. Each session consisted of 300 experimental trials in addition to those trials necessary to achieve initial alignment of the comeal reflection equipment. Each trial consisted of two phases, a "calibration" phase and a "discrimination" phase. The calibration phase, which involved only the center key, functioned to allow the experimenter to maintain the equipment in spatial calibration from trial to trial (for a detailed description, see Schrier \& Vaughan, 1973). Data analysis involved only the discrimination phase, that is, the period from the onset of the form stimuli on the side keys to the occurrence of a choice response. Recorded fixations were defined as being on the discriminative stimuli in much the same way as in the previous research, except that the size of the discriminative stimulus area in addition to its location on the recording grid was adjusted to compensate for minor day-to-day differences in alignment of the recording apparatus.

The experiment was conducted in stages, with the criteria for changing stages being five consecutive sessions of at least $90 \%$ accuracy of choice responses and apparently asymptotic visual behavior (indicated by fixation durations and either response latencies for Thad and Grady or number of scans for Klaus and Hadyn). Stage I was a simple two-choice discrimination, with one stimulus (A stimulus) indicating the side on which a choice response would be rewarded and the other (B stimulus) the nonrewarded side. In Stage II, trials on which the Stage I discrimination ( $A B$ subproblem) was presented were mixed randomly with trials on which the B stimulus was paired with a third stimulus (C stimulus), with B now rewarded and $C$ nonrewarded (BC subproblem). Thad and Grady were given a
Stage III during which a third subproblem (AC, with $\mathrm{C}$ rewarded) was mixed randomly with the others. Klaus and Hadyn were not run on Stage III but were returned to Stage I after Stage II.

\section{RESULTS}

All animals achieved criterion in Stage I and Stage II. Neither Thad nor Grady surpassed 90\% correct choice responses during Stage III, but averaged over $80 \%$ correct in the last five sessions.

Three aspects of the eye movements associated with this choice behavior were investigated: duration of the last fixation on a discriminative stimulus preceding the choice response; duration of other, earlier ("other-than-last") fixations on the discriminative stimuli; and the number of scans (shifts of fixation from one stimulus to the other). ${ }^{1}$ These data, together with choice performance and latency of choice response, for acquisition and criterion sessions of Stage I are presented in Figure 1 for Klaus and Hadyn. These latter two animals required considerably more sessions to reach criterion on the simple discrimination problem than did Thad and Grady, who required only three and two sessions to reach criterion, respectively. This difference was probably due at least in part to the different size of stimuli.

As Figure 1 shows, there were a number of changes in visual behavior during Stage I which were quite similar for the two animals, although they differed in speed of acquisition. Klaus' slower learning was associated with a strong side preference in the initial sessions. This was disrupted in Sessions 5 and 6 by use of a correction procedure during which the positive stimulus appeared only on the nonpreferred side. The initial effect of this was an increase in the duration of last fixation and latency of response in Session 5, followed in the next several sessions by a large increase in the level of scanning and a marked improvement in choice performance. After Session 10, scanning decreased, while choice performance continued to improve slightly. A similar temporal pattern of amount of scanning, that is an increase and then a decrease, was evident for Hadyn. The final level of scanning for both animals was about .5 scans per trial. (The local drop in scanning around Session 32 for Hadyn represented a transient tendency to respond to a key without looking at it). This was also the criterion level maintained during Stage $I$ by one of the other animals (Thad) that was tested. The fourth animal (Grady) scanned more frequently.

Duration of the last fixation was, as can be seen in Figure 1, comparatively constant throughout Stage I (about $400 \mathrm{msec}$ for each animal). By contrast, duration of other-than-last fixations increased from about $200 \mathrm{msec}$ initially to about $350 \mathrm{msec}$, a rather large increase in comparison with previous observations. The 

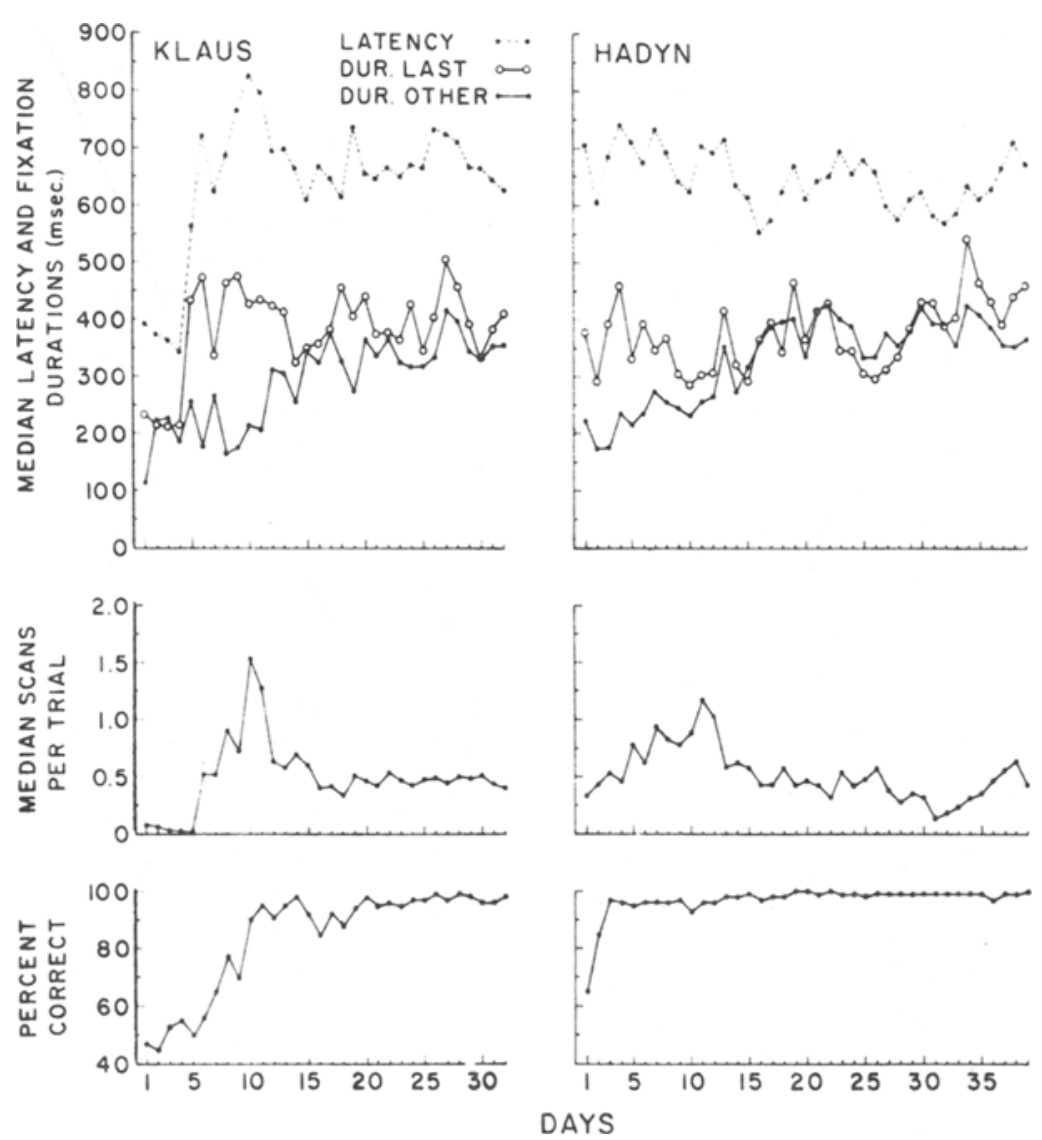

Figure 1. Median latency of choice response, median durations of last and other-than-last fixations on the discriminative stimuli, median scans, and percentage of correct responses for acquisition and criterion sessions during Stage I for Klaus and Hadyn.

reason for the increase in duration of other-than-last fixations is not clear, but it is interesting to note that this change continued until about Session 20, near when the learning curves reached their asymptotic levels. The precriterion period was too short to provide a clear picture of changes in eye movement measures for Thad and Grady.

Eye movement and latency of response data recorded during the criterion sessions of all stages for all four animals are shown in Figure 2. There were consistent changes associated with the various stages. In addition, the duration of both last and other-than-last fixations were greater for the animals tested with the small stimuli (Klaus and Hadyn) than for the animals tested with the large stimuli.

Manipulation of stimulus ambiguity in Stages II and III was clearly reflected in latency of response. Mean latency of the four animals was significantly greater during criterion sessions of Stage II than of Stage I (log transformation, $\mathrm{t}=3.85$, $\mathrm{df}=3, \mathrm{p}<.05$ ); in addition, latencies increased further for the two animals given Stage III and decreased to near their previous levels for the two animals returned to Stage I. Latency changes were, as in previous work (Schrier \& Vaughan, 1973), closely related to changes in amount of scanning. Scanning changes showed the same pattern of increases and decreases as latency, with the difference between Stage I and Stage II means again significant (square root transformation, $t=3.45$, df $=3, p<.05$ ).

The specific levels of scanning observed were in general very close to the levels predicted by a minimum frequency of observing hypothesis which assumes the following conditions: (a) the positive stimulus appears equally often on each side in a random sequence, (b) the animal searches most efficiently for the positive stimulus, and (c) the animal looks at it during its response. Under these conditions, on half the trials during Stage I, the initial fixation will be on the positive stimulus (A stimulus), and the animal will respond without scanning (0-scans trial); on the other half, the initial fixation will be on the negative stimulus (B stimulus), and the animal will shift its fixation to the other stimulus before responding (1-scan trial). Thus, the predicted minimum level of scanning during the Stage I simple discrimination is .5 scans per trial. The same predictions would hold if the animal searched most 

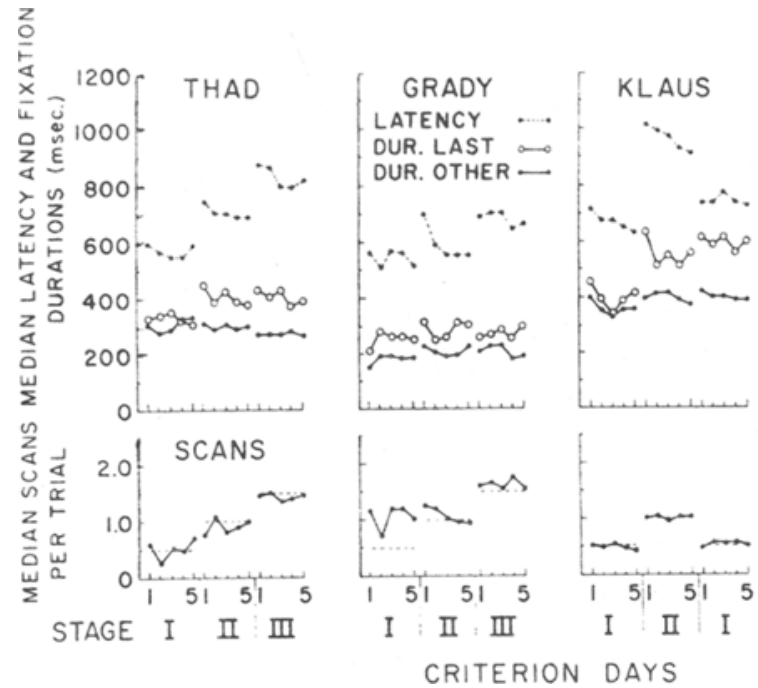

efficiently for the negative stimulus, but here, as has been the case in previous eye movement studies (Schrier \& Wing, 1973), the animal virtually always searched for the positive stimulus. Derivation of the minimum frequency of observing hypothesis prediction for scanning during Stage II is similar. The $\mathrm{AB}$ subproblem carried over from Stage I occurs on half the trials, and, as before, there should be an average of .5 scans for these trials. On the remaining trials, $\mathrm{BC}$ trials, if the $\mathrm{C}$ stimulus is viewed first, the animal has to scan once to the positive B stimulus (1-scan trial). If the B stimulus is viewed first, the animal is not able to identify it as the positive stimulus because it is ambiguous and must fixate the other stimulus to identify which is positive and then scan back in order to look at the positive stimulus during the response (2-scans tria) ${ }^{1}$. Thus, the $\mathrm{BC}$ subproblem should require an average of 1.5 scans per trial, and the average predicted amount of scanning for both Stage II subproblems combined is 1.0 scan per trial. In the Stage III problem, one scan is always necessary to identify the positive stimulus because all stimuli are ambiguous, and again, an additional .5 scans per trial to fixate it, for a total of 1.5 scans per trial. As can be seen in the lower row of graphs in Figure 2, in 11 of the 12 instances in which terminal behavioral levels were observed (the major exception being Grady's Stage I data) the observed amounts of scanning were very close to these predictions. The average terminal level of scanning by the four animals did not differ significantly from the predicted levels during either Stage I (counting either the first or second presentations of Stage $I$ in the case of Klaus and Hadyn) or Stage II. Furthermore, analysis by subproblem indicated that the average observed amounts were consistent with predictions for the individual subproblems. For example, in Stage II, the $\mathrm{AB}$ subproblem should require an average of .5 scans per trial and the $\mathrm{BC}$ subproblem, 1.5 scans per trial. The median levels for the four animals during the criterion sessions of Stage II were .57 scans per trial and 1.33 scans per trial for the $A B$ and $B C$ subproblems, respectively.

There were no significant changes in duration of other-than-last fixations after Stage I. However, mean duration of the last fixation was significantly greater during criterion sessions of Stage II than of Stage I (log transformation, $t=3.64, \mathrm{df}=3, \mathrm{p}<.05$ ). This increase in duration of last fixation occurred for the $\mathrm{BC}$ subproblem, which was introduced in Stage II, as well as the $\mathrm{AB}$ subproblem, which was carried over from Stage I. In Stage II, last fixations during the AB subproblem (in which the nonambiguous cue $A$ was positive) were significantly longer than last fixations during the $\mathrm{BC}$ subproblem (in which the ambiguous cue $B$ was positive $)(t=4.67, \mathrm{df}=3, \mathrm{p}<.02)$, although amount of scanning $(\mathrm{t}=4.92, \mathrm{df}=3, \mathrm{p}<.02)$ and latency $(t=4.67, \mathrm{df}=3, \mathrm{p}<.02)$ were significantly greater during the $\mathrm{BC}$ subproblem. There were no consistent changes in duration of the last fixation after Stage II.

Last fixations tended to be longer than other-than-last fixations throughout the experiment, and during the criterion sessions of Stage II, they were significantly longer $(t=17.50, d f=3, p<.002)$.

\section{DISCUSSION}

The amount of observing responding may be reflected in the duration of fixations, the amount of scanning, or both. In this experiment, amount of scanning approached a low level after long practice both in the simple discrimination, as seen in previous experiments, and in the subsequent ambiguous cue problems. Furthermore, the level during each stage approximated the theoretical minimum based on the assumption of an efficient search for the positive cue. Thus, the minimum frequency of observing hypothesis seems to provide an accurate description of performance in a variety of discrimination situations.

We would stress, however, that this conclusion should 
not be interpreted more broadly to refer to observing behavior in general. Observing behavior as measured by duration of other-than-last fixations increased during acquisition in Stage I for Klaus and Hadyn and remained high, and there were increases in duration of last fixations associated with succeeding stages for all animals. Thus, contrary to previous reports, it appears that lasting increases in observing behavior do accompany discrimination learning.

The idea that processing changes are reflected in duration of fixation (Gould, 1967; Neisser, 1963) finds support in the present data. Duration of last fixations were reliably longer during ambiguous cue than nonambiguous cue problems. Since last fixations were longer than preceding fixations and since the instrumental choice response occurred during the last fixation, it is likely that the duration of the last fixation reflects time to carry out this response (Schrier \& Vaughan, 1973). However, there is no reason to believe that this response time would differ for the two types of problems, since the response requirements are the same once the decision to respond is made. Hence, on the basis of the present evidence, it appears that last fixations may also reflect cognitive processing stages preceding the choice response.

The changes in duration of fixation described above as well as other changes found here indicate that this aspect of visual behavior is more labile than earlier work suggested (Schrier \& Vaughan, 1973; Schrier \& Wing, 1973). In the studies just cited, duration of fixation during color, form, and brightness discriminations typically averaged less than $210 \mathrm{msec}$. In general, the fixations were longer in the present study and were comparable to durations reported for humans performing various visual tasks (Gould \& Dill, 1969; Yarbus, 1967). Durations of fixation were also longer for the animals tested with the smaller stimuli, which, as in humans, indicates that stimulus variables affect fixation durations.

\section{REFERENCES}

D'Amato, M., Etkin, M., \& Fazzaro, J. Cue producing behavior in the Capuchin monkey during reversal, extinction, acquisition, and overtraining. Journal of the Experimental Analysis of Behavior, 1968, 11, 425-433.

Freindlich, A. Primate head restrainer using a nonsurgical technique. Journal of Applied Phy siology, 1973, 35, 934-935.

French, G. Associative problems. In A. Schrier, M. Harlow, and F. Stollnitz (Eds.) Behovior of nonhuman primates (Vol. 1). New York: Academic Press, 1965.

Gould, J. Pattern recognition and eye movement parameters. Perception \& Psychophysics, 1967, 2, 399-407.

Gould, J., \& Dill, A. Eye movement parameters and pattem discrimination. Perception \& Psychophysics, 1969, 5, 311-320.

Neisser, U. Decision time without reaction time: Experiments in visual scanning. American Journal of Psychology, 1963, 76, 376-386.

Premack, D., \& Collier, G. Duration of looking and number of brief looks as dependent variables. Psychonomic Science, $1966,4,81-82$.

Schrier, A., Povar, M., \& Vaughan, J. Measurement of eye orientation of monkeys during visual discrimination. Behavior Research Methods \& Instrumentation, 1970, 2, 55-62.

Schrier, A., Povar, M., \& Vaughan, J. Primates in eye movement research. In E. Goldsmith and J. Moor-Jankowski (Eds.), Medical primatology, 1970. Basel: Karger, 1971.

Schrier, A., \& Vaughan, J. Eye movements of monkeys during learning of color and form discrimination problems involving reversal and nonreversal shifts. Primates, 1973, 4, 161-178.

Schrier, A., \& Wing, T, Eye movements of monkeys during brightness discrimination and discrimination reversal. Animal Learning \& Behavior, 1973, 1, 145-150.

Trabasso, T.. \& Bower, G. Attention in learning: Theory and research. New York: Wiley, 1968.

White, S., \& Plum, G. Eye movement photography during children's discrimination learnirg. Journal of Experimental Child Psychology, 1964, 1, 327-338.

Yarbus, A. Eye movements and vision. New York: Plenum, 1967.

\section{NOTE}

1. For the present analyses, the last fixation on a discriminative stimulus refers only to a fixation, the recording of which was terminated by an instrumental choice response. On a small number of trials, the animals looked away from the discriminative stimulus (probably toward the food delivery device) just before the choice response was recorded. Such trials were not included in the analy sis of last fixations. A fixation on only one of the discriminative stimuli would be zero scans; a fixation on first one then the other discriminative stimulus would be one scan, etc. For purposes of measuring scans, repeated fixations on one of the discriminative stimuli with no intervening fixations on the other stimulus would be counted as only one fixation.

(Received for publication November 4, 1974; accepted January 3, 1975.) 